\title{
Clinical and prognostic heterogeneity of $C$ and D GOLD groups
}

\section{To the Editor:}

"High risk" groups for exacerbations of chronic obstructive pulmonary disease (COPD) in the 2011 Global Initiative for Chronic Obstructive Lung Disease (GOLD) proposal (i.e. groups C and D) [1] include: patients with a forced expiratory volume in $1 \mathrm{~s} \mathrm{FEV1}<50 \%$ reference and $<2$ exacerbations year ${ }^{-1}$ (subgroups C1 and D1); patients with $\geqslant 2$ exacerbations.year ${ }^{-1}$ and an FEV $1 \geqslant 50 \%$ reference (subgroups C2 and D2); and patients with both FEV $1<50 \%$ ref. and $\geqslant 2$ exacerbations.year ${ }^{-1}$ (subgroups C3 and D3) [2-5]. We hypothesised that these high-risk subgroups will differ in other clinical, functional and biological characteristics and will be associated with different long-term outcomes. We explored this hypothesis in the ECLIPSE (Evaluation of COPD Longitudinally to Identify Predictive Surrogate Endpoints) cohort [6, 7].

The design and methodology of the ECLIPSE study (www.clinicaltrials.gov with identifier number NCT00292552; GSK study code SCO104960) has been published elsewhere in detail [6]. The study was approved by the Ethics Committees from the participating centres and all participants signed their informed consent [7]. Out of the 2164 GOLD grades II-IV patients included in the ECLIPSE study, 2101 (97\%) had complete GOLD 2011 data [4] and were included in the current analysis; 1313 of them (62.5\%) were classified as groups C $(n=483,36.8 \%)$ or $D(n=830,63.2 \%)$, using the modified Medical Research Council dyspnoea score to determine high and low COPD symptoms.

These analyses are exploratory and may have low power, since they are based on a subset of the COPD subjects enrolled in ECLIPSE. Results are shown as mean \pm SD or n (\%), as appropriate. Kruskal-Wallis tests and Cochran-Mantel-Haenszel tests were used to assess differences in subject characteristics among groups. Kaplan-Meier curves were constructed to describe the occurrence of the first event for the following outcomes: moderate-to-severe exacerbations of COPD, hospitalisations for COPD exacerbation, and all-cause mortality over the study period. A p-value $<0.05$ (two sided) was considered statistically significant. No adjustments were made for multiple comparisons. SAS (version 9; SAS Institute, Inc., Cary, NC, USA) was used to conduct all analyses and figures were created via S-PLUS (TIBCO Software, Boston, MA, USA).

On the one hand, from the 483 group C patients, 336 (70\%) 62 (13\%) and 85 (18\%) were classified in the subgroups C1, C2 and C3, respectively [4]. As expected, lung function was worse in subgroups C1 and C3 (both groups had clinically comparable airflow limitation), and exacerbations prior to the start of the study were more frequent in subgroups C2 and C3 (both groups had a comparable level of exacerbation history). The remaining variables were similar across groups (table 1). However, of note is that the C2 subgroup included more females and had less emphysema than the other two C subgroups.

On the other hand, from the 830 group D patients, 522 (63\%), 72 (9\%) and 236 (28\%) were classified in subgroups D1, D2 and D3, respectively [4] (table 1). Similarly to group C patients, lung function was worse in subgroup D1 and D3 (both groups had clinically comparable airflow limitation), and previous exacerbations were more frequent in subgroups D2 and D3 (both groups had a comparable level of exacerbation history). Other variables were similar across groups, albeit the percentage of females and body mass index (BMI) was higher, and the extent of emphysema was lower, in the D2 subgroup (table 1). Of note, the 6-min walking distance (6MWD) was not different in subgroup D1 versus subgroup D2 but, at variance with the $\mathrm{C}$ subgroups, it was worse in subgroup D3.

Given that in 2013, GOLD included a new high-risk criteria (>1 hospitalisation due to COPD in the previous year) [1], we explored how this new criteria influenced the results discussed above. It caused 53 patients $(2.5 \%)$ to change from a low- to high-risk group, 26 from group A to group C, and 27 from group B to group D.

Rate of moderate-to-severe exacerbation during follow-up was higher in those patients with a history of previous exacerbations (subgroups C2/C3 and D2/D3) (table 1 and fig. 1). Time to first hospitalisation was significantly worse in subgroups C3 and D3 but similar between subgroups C1/C2 and D1/D2 (fig. 1). All-cause mortality during follow-up was different in C subgroups (highest in C3 (14\%), intermediate in C1 (9\%) and absent (0\%) in C2) but similar in D subgroups (D1 14\%, D2 11\% and D3 14\%) (fig. 1). Finally, the rate of FEV1 decline and the incidence of cardiovascular events or cancer during follow-up were not different across $\mathrm{C}$ and $\mathrm{D}$ subgroups (table 1). Both in GOLD groups $\mathrm{C}$ and $\mathrm{D}$, age, FEV $1 \%$ 
TABLE 1 The main characteristics and outcomes for the three high-risk $C$ and $D$ subgroups

\begin{tabular}{|c|c|c|c|c|c|c|c|c|c|c|c|c|}
\hline & \multirow[t]{2}{*}{ C1 } & \multirow[t]{2}{*}{ C2 } & \multirow[t]{2}{*}{ C3 } & \multicolumn{3}{|c|}{ p-value } & \multirow[t]{2}{*}{ D1 } & \multirow[t]{2}{*}{ D2 } & \multirow[t]{2}{*}{ D3 } & \multicolumn{3}{|c|}{$\mathrm{p}$-value } \\
\hline & & & & $\begin{array}{c}\text { C1 versus } \\
\text { C2 }\end{array}$ & $\begin{array}{c}\text { C1 versus } \\
\text { C3 }\end{array}$ & $\begin{array}{c}\text { C2 versus } \\
\text { C3 }\end{array}$ & & & & $\begin{array}{c}\text { D1 versus } \\
\text { D2 }\end{array}$ & $\begin{array}{c}\text { D1 versus } \\
\text { D3 }\end{array}$ & $\begin{array}{c}\text { D2 versus } \\
\text { D3 }\end{array}$ \\
\hline Patients & $336(70 \%)$ & $62(13 \%)$ & $85(18 \%)$ & & & & $522(63 \%)$ & $72(9 \%)$ & $236(28 \%)$ & & & \\
\hline \multicolumn{13}{|l|}{ Demographics } \\
\hline Age years & $63.2 \pm 7.2$ & $61.8 \pm 7.7$ & $62.3 \pm 6.9$ & 0.228 & 0.215 & 0.866 & $63.7 \pm 7.0$ & $63.5 \pm 7.1$ & $63.5 \pm 6.6$ & 0.963 & 0.577 & 0.72 \\
\hline Female & $95(28 \%)$ & $27(44 \%)$ & $27(32 \%)$ & 0.017 & 0.527 & 0.145 & $147(28 \%)$ & $41(57 \%)$ & $92(39 \%)$ & $<0.001$ & 0.003 & 0.007 \\
\hline $\mathrm{BMI} \mathrm{kg} \cdot \mathrm{m}^{-2}$ & $25.1 \pm 5.0$ & $25.9 \pm 4.7$ & $25.2 \pm 5.0$ & 0.299 & 0.961 & 0.444 & $26.3 \pm 6.0$ & $29.0 \pm 7.3$ & $26.3 \pm 5.7$ & 0.003 & 0.826 & 0.007 \\
\hline $\mathrm{FFMI} \mathrm{kg} \cdot \mathrm{m}^{-2}$ & $16.7 \pm 2.5$ & $17.1 \pm 2.6$ & $16.6 \pm 2.4$ & 0.365 & 0.895 & 0.416 & $17.0 \pm 2.8$ & $17.9 \pm 3.3$ & $17.0 \pm 2.9$ & 0.027 & 0.74 & 0.028 \\
\hline Smoking pack-years & $45.3 \pm 22.7$ & $46.1 \pm 24.1$ & $44.2 \pm 24.6$ & 0.948 & 0.439 & 0.552 & $51.5 \pm 26.7$ & $50.3 \pm 33.8$ & $50.6 \pm 27.9$ & 0.201 & 0.696 & 0.356 \\
\hline Period smoking years & $39.7 \pm 10.4$ & $39.9 \pm 10.0$ & $39.4 \pm 7.7$ & 0.769 & 0.413 & 0.347 & $40.2 \pm 9.6$ & $40.8 \pm 8.3$ & $40.8 \pm 10.0$ & 0.878 & 0.305 & 0.65 \\
\hline Current smoker & $143(43 \%)$ & $32(52 \%)$ & $32(38 \%)$ & 0.188 & 0.412 & 0.093 & $167(32 \%)$ & $26(36 \%)$ & 74 (31\%) & 0.485 & 0.862 & 0.451 \\
\hline \multicolumn{13}{|l|}{ Symptoms } \\
\hline mMRC dyspnoea score & $0.8 \pm 0.4$ & $0.8 \pm 0.4$ & $0.8 \pm 0.4$ & 0.911 & 0.46 & 0.519 & $2.5 \pm 0.7$ & $2.5 \pm 0.7$ & $2.7 \pm 0.8$ & 0.932 & 0.026 & 0.245 \\
\hline SGRQ-C total score & $43.2 \pm 17.0$ & $41.8 \pm 14.9$ & $51.9 \pm 15.2$ & 0.597 & $<0.001$ & $<0.001$ & $60.2 \pm 15.8$ & $65.3 \pm 15.3$ & $65.5 \pm 14.4$ & 0.009 & $<0.001$ & 0.933 \\
\hline Moderate-to-severe ECOPD $\#$ & $0.3 \pm 0.5$ & $2.5 \pm 1.0$ & $2.8 \pm 1.5$ & $<0.001$ & $<0.001$ & 0.133 & $0.4 \pm 0.5$ & $2.8 \pm 1.1$ & $2.9 \pm 1.2$ & $<0.001$ & $<0.001$ & 0.423 \\
\hline CESD total score & $9.2 \pm 8.2$ & $10.1 \pm 7.4$ & $10.0 \pm 8.9$ & 0.166 & 0.558 & 0.552 & $12.5 \pm 9.2$ & $15.3 \pm 10.5$ & $15.3 \pm 10.0$ & 0.033 & $<0.001$ & 0.863 \\
\hline Chronic bronchitis & $126(38 \%)$ & $28(45 \%)$ & $28(33 \%)$ & 0.256 & 0.436 & 0.133 & $195(37 \%)$ & $28(39 \%)$ & $95(40 \%)$ & 0.801 & 0.447 & 0.836 \\
\hline \multicolumn{13}{|l|}{ Physiology" } \\
\hline $\mathrm{FEV}_{1}+\%$ pred & $39.7 \pm 7.6$ & $63.7 \pm 8.7$ & $36.3 \pm 8.5$ & $<0.001$ & $<0.001$ & $<0.001$ & $35.1 \pm 8.7$ & $60.2 \pm 9.3$ & $35.1 \pm 8.5$ & $<0.001$ & 0.969 & $<0.001$ \\
\hline FEV $1 \%$ reversibility & $10.1 \pm 13.5$ & $8.7 \pm 9.7$ & $12.1 \pm 15.7$ & 0.517 & 0.491 & 0.259 & $10.3 \pm 14.7$ & $9.6 \pm 10.9$ & $10.4 \pm 15.2$ & 0.835 & 0.97 & 0.852 \\
\hline $\mathrm{FVC}^{+} \%$ pred & $81.4 \pm 17.4$ & $99.5 \pm 16.5$ & $81.3 \pm 18.9$ & $<0.001$ & 0.938 & $<0.001$ & $74.8 \pm 17.0$ & $96.0 \pm 15.5$ & $78.1 \pm 19.1$ & $<0.001$ & 0.025 & $<0.001$ \\
\hline RV \% pred & $175.1 \pm 48.8$ & $147.0 \pm 36.1$ & $184.1 \pm 50.5$ & 0.038 & 0.271 & 0.023 & $180.9 \pm 47.4$ & $122.6 \pm 29.3$ & $189.7 \pm 56.3$ & $<0.001$ & 0.589 & $<0.001$ \\
\hline TLC \% pred & $121.4 \pm 16.5$ & $118.2 \pm 14.7$ & $122.8 \pm 18.9$ & 0.612 & 0.532 & 0.351 & $121.1 \pm 18.4$ & $110.2 \pm 15.3$ & $123.8 \pm 19.4$ & 0.025 & 0.726 & 0.014 \\
\hline FRC $\%$ pred & $157.0 \pm 29.3$ & $137.8 \pm 27.3$ & $162.2 \pm 34.1$ & 0.017 & 0.381 & 0.011 & $160.9 \pm 35.1$ & $124.2 \pm 26.0$ & $161.7 \pm 36.7$ & $<0.001$ & 0.935 & $<0.001$ \\
\hline $\mathrm{SaO}_{2} \%$ & $94.4 \pm 3.0$ & $95.6 \pm 2.2$ & $93.7 \pm 4.4$ & $<0.001$ & 0.188 & $<0.001$ & $93.6 \pm 3.2$ & $95.1 \pm 2.0$ & $93.7 \pm 3.3$ & $<0.001$ & 0.5 & $<0.001$ \\
\hline 6MWD m & $405 \pm 108$ & $446 \pm 97$ & $394 \pm 97$ & 0.016 & 0.515 & 0.011 & $313 \pm 117$ & $320 \pm 92$ & $289 \pm 111$ & 0.633 & 0.007 & 0.033 \\
\hline BODE index & $2.8 \pm 1.0$ & $0.8 \pm 0.8$ & $3.0 \pm 1.0$ & $<0.001$ & 0.192 & $<0.001$ & $5.1 \pm 1.5$ & $3.3 \pm 1.2$ & $5.5 \pm 1.6$ & $<0.001$ & 0.014 & $<0.001$ \\
\hline \multicolumn{13}{|l|}{ Imaging } \\
\hline$\%$ LAA -950 HU & $19.9 \pm 11.1$ & $10.7 \pm 8.2$ & $21.3 \pm 13.0$ & $<0.001$ & 0.429 & $<0.001$ & $22.9 \pm 13.2$ & $15.2 \pm 9.8$ & $24.0 \pm 11.9$ & $<0.001$ & 0.178 & $<0.001$ \\
\hline \multicolumn{13}{|l|}{ Systemic inflammation } \\
\hline WBC $10^{9} \cdot \mathrm{L}^{-1}$ & $7.6(2.5)$ & $7.6(2.3)$ & $7.4(2.6)$ & 0.584 & 0.312 & 0.749 & $7.8(2.6)$ & $7.7(2.7)$ & $8.0(2.6)$ & 0.779 & 0.106 & 0.264 \\
\hline Neutrophils $10^{9} \cdot \mathrm{L}^{-1}$ & $4.9(2.3)$ & $4.4(1.8)$ & $4.9(2.1)$ & 0.105 & 0.467 & 0.376 & $5.2(2.2)$ & $4.8(2.5)$ & $5.2(2.4)$ & 0.263 & 0.198 & 0.075 \\
\hline Neutrophils \% & $65.5(11.2)$ & $62.1(11.4)$ & $66.3(11.2)$ & $<0.001$ & 0.922 & 0.006 & $66.9(11.3)$ & $65.1(12.7)$ & $67.0(10.5)$ & 0.05 & 0.865 & 0.047 \\
\hline Eosinophils $10^{9} \cdot \mathrm{L}^{-1}$ & $0.2(0.2)$ & $0.2(0.2)$ & $0.2(0.2)$ & 0.481 & 0.253 & 0.164 & $0.2(0.2)$ & $0.2(0.1)$ & $0.2(0.2)$ & 0.994 & 0.056 & 0.217 \\
\hline Eosinophils \% & $2.6(2.3)$ & $3.1(2.7)$ & $2.3(2.9)$ & 0.342 & 0.446 & 0.256 & $2.3(1.9)$ & $2.1(1.4)$ & $2.4(2.6)$ & 0.943 & 0.187 & 0.486 \\
\hline $\mathrm{hsCRP} \mathrm{mg} \cdot \mathrm{L}^{-1}$ & $2.5(4.2)$ & $2.7(5.7)$ & $4.0(5.9)$ & 0.478 & 0.024 & 0.031 & $3.7(7.5)$ & $3.9(6.3)$ & $4.5(7.8)$ & 0.615 & 0.028 & 0.381 \\
\hline $\mathrm{IL}-6 \mathrm{pg} \cdot \mathrm{mL}^{-1}$ & $1.4(2.1)$ & $1.2(1.7)$ & $1.4(2.1)$ & 0.096 & 0.993 & 0.184 & $1.8(2.7)$ & $2.1(2.4)$ & $1.8(2.9)$ & 0.545 & 0.67 & 0.507 \\
\hline $\mathrm{IL}-8 \mathrm{pg} \cdot \mathrm{mL}^{-1}$ & $6.4(9.5)$ & $6.6(9.3)$ & $6.7(8.6)$ & 0.641 & 0.812 & 0.888 & $7.0(10.6)$ & $8.8(10.4)$ & $7.5(9.1)$ & 0.315 & 0.381 & 0.54 \\
\hline Fibrinogen $\mathrm{mg} \cdot \mathrm{dL}^{-1}$ & $442.0(125.0)$ & $436.5(152.5)$ & $466.0(132.0)$ & 0.528 & 0.04 & 0.071 & $470.0(147.5)$ & $457.3(106.0)$ & $483.0(134.0)$ & 0.522 & 0.029 & 0.032 \\
\hline$T N F-\alpha n g \cdot m L^{-1}$ & $2.4(0.0)$ & $2.4(51.4)$ & $2.4(4.8)$ & 0.001 & 0.486 & 0.036 & $2.4(2.7)$ & $2.4(7.4)$ & $2.4(0.0)$ & 0.681 & 0.335 & 0.313 \\
\hline $\mathrm{CC}-16 \mathrm{ng} \cdot \mathrm{mL}^{-1}$ & $5.1(3.0)$ & $4.6(3.5)$ & $5.4(4.2)$ & 0.116 & 0.788 & 0.154 & $4.8(3.4)$ & $4.7(3.4)$ & $4.9(3.4)$ & 0.168 & 0.906 & 0.191 \\
\hline $\mathrm{CCL}-18 \mathrm{ng} \cdot \mathrm{mL}^{-1}$ & $100.0(48.8)$ & $103.1(48.2)$ & $98.9(49.6)$ & 0.24 & 0.318 & 0.702 & $107.1(59.0)$ & $109.9(56.9)$ & $114.0(54.7)$ & 0.408 & 0.112 & 0.838 \\
\hline $\mathrm{SPD} n \mathrm{ng} \cdot \mathrm{mL}^{-1}$ & $118.4(91.2)$ & $120.4(58.9)$ & $133.1(95.0)$ & 0.663 & 0.108 & 0.113 & $121.3(85.0)$ & $122.8(99.2)$ & $115.8(87.0)$ & 0.763 & 0.167 & 0.665 \\
\hline
\end{tabular}




\begin{tabular}{|c|c|c|c|c|c|c|c|c|c|c|c|c|}
\hline & \multirow[t]{2}{*}{ c1 } & \multirow[t]{2}{*}{$\mathrm{C2}$} & \multirow[t]{2}{*}{ C3 } & \multicolumn{3}{|c|}{$\mathrm{p}$-value } & \multirow[t]{2}{*}{ D1 } & \multirow[t]{2}{*}{ D2 } & \multirow[t]{2}{*}{ D3 } & \multicolumn{3}{|c|}{$\mathrm{p}$-value } \\
\hline & & & & $\begin{array}{c}\text { C1 versus } \\
\text { C2 }\end{array}$ & $\begin{array}{l}\text { C1 versus } \\
\text { C3 }\end{array}$ & $\begin{array}{l}\text { C2 versus } \\
\text { C3 }\end{array}$ & & & & $\begin{array}{l}\text { D1 versus } \\
\text { D2 }\end{array}$ & $\begin{array}{l}\text { D1 versus } \\
\text { D3 }\end{array}$ & $\begin{array}{l}\text { D2 versus } \\
\text { D3 }\end{array}$ \\
\hline \multicolumn{13}{|l|}{ Concomitant medications } \\
\hline Medications containing ICS & $241(72 \%)$ & $44(71 \%)$ & 79 (93\%) & 0.903 & $<0.001$ & $<0.001$ & $421(81 \%)$ & $63(88 \%)$ & $218(92 \%)$ & 0.161 & $<0.001$ & 0.201 \\
\hline Tiotropium & $146(43 \%)$ & $23(37 \%)$ & $50(59 \%)$ & 0.353 & 0.011 & 0.01 & $271(52 \%)$ & $43(60 \%)$ & 135 (57\%) & 0.214 & 0.177 & 0.705 \\
\hline Statins & $69(21 \%)$ & $12(19 \%)$ & $15(18 \%)$ & 0.832 & 0.552 & 0.792 & $126(24 \%)$ & $17(24 \%)$ & $44(19 \%)$ & 0.922 & 0.093 & 0.355 \\
\hline \multicolumn{13}{|l|}{ Comorbidities $^{\S}$} \\
\hline Heart trouble & $64(19 \%)$ & $10(16 \%)$ & $15(18 \%)$ & 0.561 & 0.839 & 0.735 & $151(30 \%)$ & $20(28 \%)$ & $63(28 \%)$ & 0.796 & 0.551 & 0.914 \\
\hline Hypertension & $113(35 \%)$ & $17(30 \%)$ & $23(29 \%)$ & 0.477 & 0.316 & 0.892 & $203(41 \%)$ & $28(42 \%)$ & $93(41 \%)$ & 0.797 & 0.922 & 0.854 \\
\hline Angina & $25(8 \%)$ & $5(8 \%)$ & $8(10 \%)$ & 0.936 & 0.536 & 0.71 & $47(10 \%)$ & $11(16 \%)$ & $32(14 \%)$ & 0.099 & 0.09 & 0.637 \\
\hline Heart attack & $16(5 \%)$ & 0 & $6(7 \%)$ & 0.076 & 0.381 & 0.03 & $46(9 \%)$ & $10(15 \%)$ & $24(10 \%)$ & 0.138 & 0.548 & 0.331 \\
\hline Stroke & $9(3 \%)$ & $4(7 \%)$ & $3(4 \%)$ & 0.124 & 0.678 & 0.409 & $17(3 \%)$ & $5(7 \%)$ & $7(3 \%)$ & 0.107 & 0.843 & 0.117 \\
\hline Heart failure & $13(4 \%)$ & $1(2 \%)$ & $5(6 \%)$ & 0.364 & 0.396 & 0.184 & $48(10 \%)$ & $5(8 \%)$ & $15(7 \%)$ & 0.609 & 0.217 & 0.812 \\
\hline Arrhythmia & $33(10 \%)$ & $5(8 \%)$ & $10(12 \%)$ & 0.671 & 0.617 & 0.481 & $61(12 \%)$ & $12(19 \%)$ & $30(14 \%)$ & 0.163 & 0.701 & 0.298 \\
\hline Osteoporosis & $38(12 \%)$ & $9(16 \%)$ & $13(16 \%)$ & 0.4 & 0.305 & 0.967 & $57(12 \%)$ & $12(17 \%)$ & $49(23 \%)$ & 0.18 & $<0.001$ & 0.351 \\
\hline Osteoarthritis & $29(9 \%)$ & $12(22 \%)$ & $13(17 \%)$ & 0.005 & 0.049 & 0.456 & $53(11 \%)$ & $25(36 \%)$ & $34(16 \%)$ & $<0.001$ & 0.056 & $<0.001$ \\
\hline Rheumatoid arthritis & $11(3 \%)$ & $2(4 \%)$ & $4(5 \%)$ & 0.902 & 0.498 & 0.729 & $12(2 \%)$ & $3(5 \%)$ & $7(3 \%)$ & 0.304 & 0.588 & 0.563 \\
\hline Inflammatory bowel disorder & $10(3 \%)$ & $2(3 \%)$ & $5(6 \%)$ & 0.878 & 0.185 & 0.47 & $24(5 \%)$ & $8(12 \%)$ & $10(4 \%)$ & 0.019 & 0.868 & 0.031 \\
\hline Diabetes & $22(7 \%)$ & 0 & $5(6 \%)$ & 0.042 & 0.847 & 0.056 & $60(12 \%)$ & $11(15 \%)$ & $21(9 \%)$ & 0.356 & 0.287 & 0.123 \\
\hline Peptic ulcer & $36(11 \%)$ & $9(16 \%)$ & $5(6 \%)$ & 0.285 & 0.191 & 0.061 & $47(9 \%)$ & $8(11 \%)$ & $28(12 \%)$ & 0.587 & 0.21 & 0.821 \\
\hline Reflux/heartburn & $65(20 \%)$ & $19(32 \%)$ & $17(20 \%)$ & 0.032 & 0.912 & 0.106 & $114(23 \%)$ & $34(49 \%)$ & $67(30 \%)$ & $<0.001$ & 0.036 & 0.003 \\
\hline $\begin{array}{l}\text { Depression } \\
\text { requiring treatment }\end{array}$ & $46(14 \%)$ & $9(15 \%)$ & $13(15 \%)$ & 0.814 & 0.734 & 0.961 & $84(16 \%)$ & 19 (27\%) & $51(22 \%)$ & 0.033 & 0.051 & 0.457 \\
\hline Anxiety/panic attacks & $37(11 \%)$ & $8(14 \%)$ & $11(13 \%)$ & 0.566 & 0.598 & 0.927 & $97(19 \%)$ & $23(32 \%)$ & $58(26 \%)$ & 0.01 & 0.049 & 0.267 \\
\hline \multicolumn{13}{|l|}{ 3-year follow-up } \\
\hline Mortality rate & $29(9 \%)$ & 0 & $12(14 \%)$ & 0.016 & 0.128 & 0.002 & $72(14 \%)$ & $8(11 \%)$ & $34(14 \%)$ & 0.532 & 0.822 & 0.476 \\
\hline $\begin{array}{l}\text { Moderate-to-severe ECOPD rate } \\
\text { per person-year }\end{array}$ & $1.1(1.2 \%)$ & $1.6(1.2 \%)$ & $2.1(1.8 \%)$ & $<0.001$ & $<0.001$ & 0.451 & $1.2(1.4 \%)$ & $2.1(1.7 \%)$ & $2.6(1.8 \%)$ & $<0.001$ & $<0.001$ & 0.018 \\
\hline Rate of FEV1 decline $\mathrm{mL} \cdot \mathrm{year}^{-1}$ & $-28.0 \pm 47.6$ & $-38.1 \pm 44.3$ & $-33.5 \pm 36.8$ & 0.15 & 0.426 & 0.559 & $-32.8 \pm 34.5$ & $-31.0 \pm 42.6$ & $-30.1 \pm 32.2$ & 0.363 & 0.861 & 0.426 \\
\hline $\mathrm{CV}$ events & $20(6 \%)$ & $4(6 \%)$ & $6(7 \%)$ & 0.88 & 0.705 & 0.886 & $29(6 \%)$ & $2(3 \%)$ & $19(8 \%)$ & 0.321 & 0.192 & 0.121 \\
\hline Lung cancer & $3 \mid<1 \%)$ & $1(2 \%)$ & 0 & 0.602 & 0.383 & 0.242 & $3(<1 \%)$ & $1(1 \%)$ & $1[<1 \%)$ & 0.429 & 0.791 & 0.373 \\
\hline
\end{tabular}

Data are presented as $\mathrm{n}(\%)$, mean $\pm \mathrm{SD}$ and median (interquartile range), unless otherwise stated. C1/D1: patients with forced expiratory volume in $1 \mathrm{~s}$ (FEV 1 ) <50\% reference, $<2$ exacerbations.year ${ }^{-1}$ and low (C1) or high (D1) symptoms; C2/D2 patients with FEV1 $\geqslant 50 \%$ ref., $\geqslant 2$ exacerbations.year ${ }^{-1}$ and low (C2) or high (D2) symptoms; C3/D3: patients with both FEV $1<50 \%$ ref., $\geqslant 2$ exacerbations.year ${ }^{-1}$ and low (C3) or high (D3) symptoms. Lung volumes were only assessed in a subset of the ECLIPSE cohort at select sites, therefore, $~ 30 \%$ of group C patients and $21 \%$ of group D patients had lung volume data. BMI: body mass index; FFMI: fat-free mass index; mMRC: modified Medical Research Council; SGRQ-C: St George's Respiratory Questionnaire for chronic obstructive pulmonary disease patients (COPD); ECOPD: exacerbation of COPD. CESD: Center for Epidemiological Studies Depression Scale; FVC: forced vital capacity; RV: residual volume; TLC: total lung capacity; FRC: functional residual capacity; $\mathrm{SaO}_{2}$ : arterial oxygen saturation; 6MWD: 6-min walking distance: BODE: BMI, airflow obstruction, dyspnoea, exercise capacity; \% LAA: percentage of low attenuation areas (i.e. emphysema) in the computed tomography; WBC: white blood cells; hsCRP: high-sensitivity C-reactive protein; IL: interleukin; TNF- $\alpha$ : tumour necrosis factor- $\alpha$; CC-16: serum club cell secretory protein; CCL-18: chemokine C-C ligand 18; SPD: surfactant protein D; ICS: inhaled corticosteroids. \#: 12 months prior to baseline; " : lung volumes were only assessed in a subset of the ECLIPSE cohort at select sites, therefore, $\sim 30 \%$ of group $C$ patients and $21 \%$ of group D patients had lung volume data; ${ }^{+}$: post-bronchodilator; ${ }^{\S}$ : based on patient self-report. 

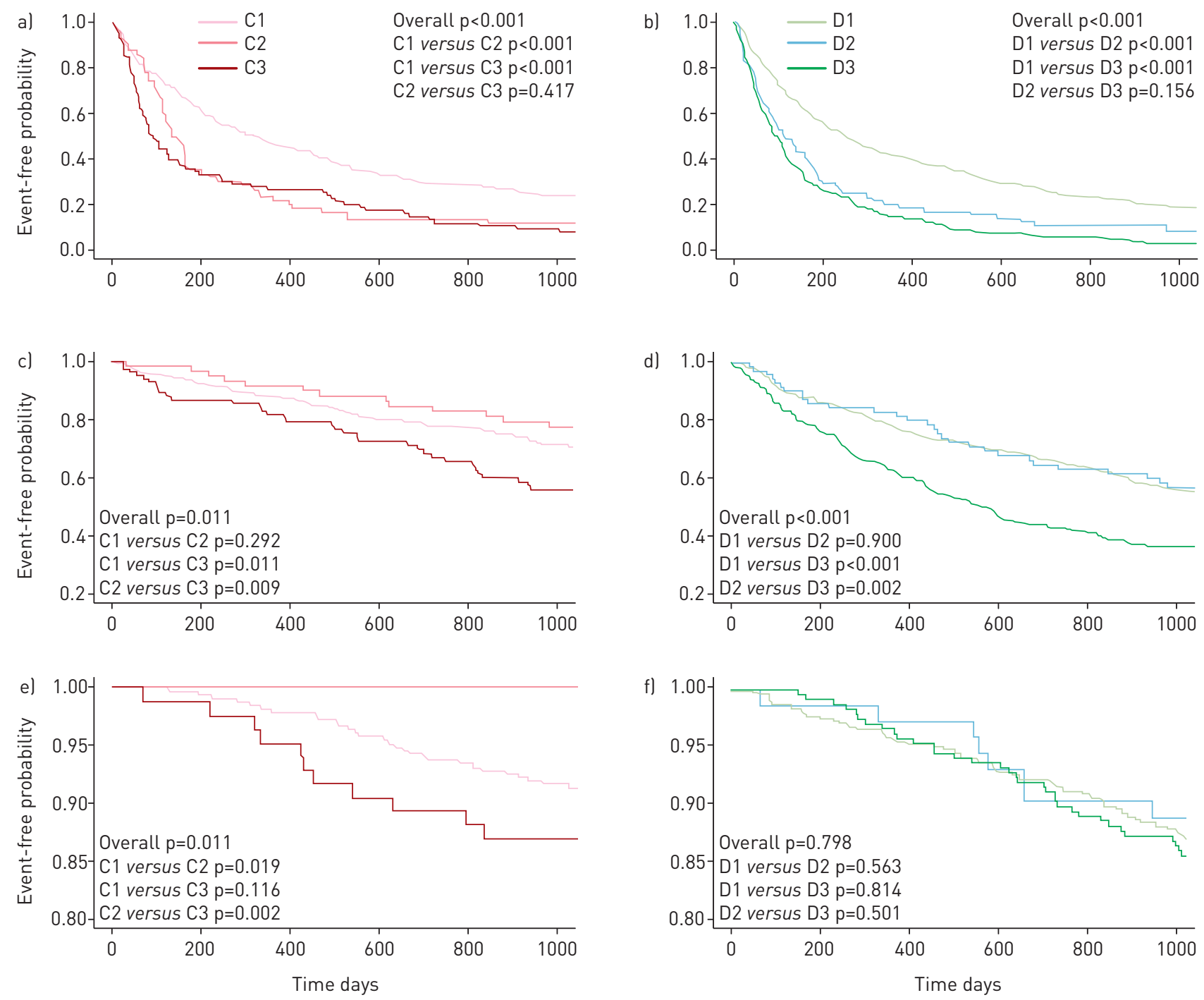

FIGURE 1 Kaplan-Meier curves for time to: $\mathrm{a}$ and b) moderate-to-severe exacerbations; $\mathrm{c}$ and d) time to hospitalisation for chronic obstructive pulmonary disease exacerbation; and e and f) all-cause mortality during follow-up in subgroups C1-C3 (a, c and e) and subgroups D1-3 (c, d and e).

predicted, 6MWD and the BMI, severity of airflow limitation, dyspnoea, exercise capacity (BODE) index were significantly associated with mortality. Similar factors, plus history of previous exacerbations, were significantly associated with hospitalisations in the two groups.

These results constitute one of the most detailed datasets of information on the characteristics and relationships with clinically relevant outcomes of the $\mathrm{C}$ and D GOLD subgroups available to date $[2,3]$. Apart from the expected differences in FEV1 and exacerbation rates in these different subgroups, it is of note that most variables were similar across them (table 1). However, a notable exception was that subgroups C2 and D2 had a higher prevalence of females (also noted by previous studies $[3,8]$ ) and a lower severity of emphysema (table 1).

Our results confirmed previous observations indicating that a $\mathrm{FEV}_{1}<50 \%$ reference alone (subgroups $\mathrm{C} 1$, D1) was the most frequent reason ( $75 \%$ of patients) to classify them as group C or D [2-4]. This may have therapeutic implications since some $\mathrm{C} 1$ and D1 subgroup patients may benefit, mostly, from bronchodilator therapy, whereas $\mathrm{C} 2 / \mathrm{C} 3$ and D2/D3 subgroups are likely to benefit most from the addition of anti-inflammatory therapy to reduce the risk of future exacerbations [9].

That exacerbations were more frequent in those subgroups defined as being at a high risk of an exacerbation, exclusively (subgroups C2 and D2) or partially (subgroups C3 and D3), by their previous history of frequent exacerbations (fig. 1) can be expected, since the strongest predictor of future exacerbations is the previous exacerbation rate [8]. In fact, LANGE et al. [3] reported similar findings. Likewise, that the time to hospitalisation was significantly shorter in the C3 and D3 subgroups, but similar in the C1/C2 and D1/D2 subgroups (fig. 1), likely reflects the co-occurrence of two known risk factors for hospitalisation (i.e. severe 
airflow limitation and frequent exacerbations) [10]. By contrast, it was of interest that all-cause mortality was highest in $\mathrm{C} 3$, intermediate in $\mathrm{C} 1$ and null in $\mathrm{C} 2$, whereas it was similar in D1, D2 and D3 (fig. 1f). Differences between $\mathrm{C}$ and $\mathrm{D}$ subgroups possibly reflect a more severe disease in the latter. On the other hand, the zero mortality during follow-up in the subgroup C2 patients is probably clinically relevant, although this could be due to the small sample size of this subgroup. Also of interest was the observation that the rate of exacerbations during follow-up was not different in subgroups $\mathrm{C} 1$ versus D1, C2 versus D2 or C3 versus $\mathrm{D} 3$, at variance with the rate of hospitalisation and mortality (except for subgroup C3 versus subgroup D3). Finally, as previously reported in the entire C and D groups [4], FEV1 decline and the incidence of cardiovascular events or cancer during follow-up was similar across subgroups (table 1).

Several potential limitations of this analysis deserve comment. First, patients in the ECLIPSE study were treated according to their local physician, so these observations are not representative of a naive COPD population. Likewise, there were treatment differences across groups, and these might have influenced outcomes by indication. Finally, most patients in the ECLIPSE study were recruited from referral centres, so results may not reflect COPD in the general population.

In summary, these results provide extensive information to better delineate the heterogeneity, both cross-sectional and longitudinally, of the C and D GOLD subgroups, which may be relevant for the design of new research aimed at optimising treatment in these high-risk COPD patients. highly heterogeneous http://ow.ly/LTMOv

Alvar Agustí $^{1}$, Stephen Rennard ${ }^{2}$, Lisa D. Edwards ${ }^{3}$, William MacNee ${ }^{4}$, Emiel Wouters ${ }^{5}$, Bruce Miller ${ }^{6}$, Ruth Tal-Singer ${ }^{6}$, Hana Mullerova ${ }^{7}$ and Bartolomé Celli ${ }^{8}$ on behalf of the Evaluation of COPD Longitudinally to Identify Predictive Surrogate Endpoints (ECLIPSE) investigators

${ }^{1}$ Thorax Institute, Hospital Clinic, IDIBAPS, University of Barcelona and CIBER Enfermedades Respiratorias (CIBERES), Barcelona, Spain. ${ }^{2}$ Dept of Pulmonary and Critical Care Medicine, University of Nebraska Medical Center, Omaha, NE, USA. ${ }^{3}$ Clinical Statistics, GlaxoSmithKline R\&D, Research Triangle Park, NC, USA. ${ }^{4}$ ELEGI Labs, University of Edinburgh, Edinburgh, UK. ${ }^{5}$ Dept of Respiratory Medicine, Maastricht University Medical Center, Maastricht, The Netherlands. ${ }^{6}$ Respiratory Therapy Area Unit, GlaxoSmithKline R\&D, King of Prussia, PA, USA. ${ }^{7}$ Worldwide Epidemiology, GlaxoSmithKline R\&D, Uxbridge, England. ${ }^{8}$ Dept of Respiratory Medicine, Brigham and Women's Hospital, Boston, MA, USA.

Correspondence: Alvar Agustí, Institut del Tòrax. Hospital Clínic, Universitat de Barcelona, Villarroel 170, Escala 3, Planta 5, 08036 Barcelona, Spain. E-mail: alvar.agusti@clinic.ub.es.

Received: Jan 222015 | Accepted after revision: March 302015

Support statement: This study was sponsored by GlaxoSmithKline. Funding information for this article has been deposited with FundRef.

Conflict of interest: Disclosures can be found alongside the online version of this article at erj.ersjournals.com

Acknowledgements: Authors thank all participants for their willingness to contribute to advance medical science in the field of COPD.

\section{References}

1 Vestbo J, Hurd SS, Agusti AG, et al. Global strategy for the diagnosis, management and prevention of chronic obstructive pulmonary disease: GOLD executive summary. Am J Respir Crit Care Med 2013; 187: 347-365.

2 Han MK, Mullerova H, Curran-Everett D, et al. GOLD 2011 disease severity classification in COPDGene: a prospective cohort study. Lancet Respir Med 2013; 1: 43-50.

3 Lange P, Marott JL, Vestbo J, et al. Prediction of the clinical course of chronic obstructive pulmonary disease, using the new GOLD classification. Am J Respir Crit Care Med 2012; 186: 975-981.

4 Agusti A, Edwards L, Celli B, et al. Characteristics, stability and outcomes of the GOLD 2011 COPD groups in the ECLIPSE cohort. Eur Respir J 2013; 42: 636-646.

5 Yusen RD. Evolution of the GOLD documents for the diagnosis, management, and prevention of chronic obstructive pulmonary disease: controversies and questions. Am J Respir Crit Care Med 2013; 188: 4-5.

6 Vestbo J, Anderson W, Coxson HO, et al. Evaluation of COPD Longitudinally to Identify Predictive Surrogate End-points (ECLIPSE). Eur Respir J 2008; 31: 869-873.

7 Agusti A, Calverley P, Celli B, et al. Characterisation of COPD heterogeneity in the ECLIPSE cohort. Respir Res 2010; 11: 122 .

8 Hurst JR, Vestbo J, Anzueto A, et al. Susceptibility to exacerbation in chronic obstructive pulmonary disease. New Engl J Med 2010; 363: 1128-1138.

9 Agusti A, Fabbri LM. Inhaled steroids in COPD: when should they be used? Lancet Respir Med 2014; 2: 869-871.

10 Prieto-Centurion V, Markos MA, Ramey NI, et al. Interventions to reduce rehospitalizations after chronic obstructive pulmonary disease exacerbations: a systematic review. Ann Am Thorac Soc 2014; 11: 417-424. 